\title{
40-Gb/s all-optical digital 4-bit priority encoder employing cross-gain modulation in semiconductor optical amplifiers
}

\author{
LEI Lei, DONG JianJi, ZHANG Yin, YU Yu \& ZHANG XinLiang* \\ Wuhan National Laboratory for Optoelectronics and the School of Optoelectronic Science and Engineering, Huazhong University of Science and \\ Technology, Wuhan 430074, China
}

Received October 14, 2011; accepted November 2, 2011

\begin{abstract}
High-speed all-optical logic circuits have attracted much attention because of their important roles in signal processing in next-generation optical networks. The digital encoder is widely used in binary calculation, multiplexing, demultiplexing, address recognition and data encryption. A priority encoder allows the existence of multiple valid inputs simultaneously, identifies the priority of the request signals and encodes the priority. We propose and experimentally demonstrate an all-optical 4-bit priority encoder for return-to-zero signals at $40 \mathrm{Gbit} / \mathrm{s}$ based on cross-gain modulation in semiconductor optical amplifiers. Detuning filters after semiconductor optical amplifiers are employed to improve the output performance. Correct logic bit sequences and clear open eye patterns with extinction ratios exceeding $10 \mathrm{~dB}$ are achieved.
\end{abstract}

all-optical logic gates, delay interferometers, semiconductor optical amplifier

Citation: Lei L, Dong J J, Zhang Y, et al. 40-Gb/s all-optical digital 4-bit priority encoder employing cross-gain modulation in semiconductor optical amplifiers. Chin Sci Bull, 2012, 57: 1204-1208, doi: 10.1007/s11434-011-4957-2

High-speed all-optical logic circuits will be widely used in next-generation optical networks because of their important roles in signal processing such as address recognition, data encryption and label swapping. Recently, various basic logic gates have been demonstrated with different nonlinear devices [1-3]. Meanwhile, many more complex all-optical logic circuits have also been reported. For combinational logic functions, a 10-Gb/s all-optical half-adder with interferometric semiconductor optical amplifier (SOA) gates has been demonstrated [4], a simultaneous optical digital halfsubtractor and adder using SOAs combined with a waveguide made from periodically poled lithium niobate has been proposed [5], an all-optical encoder and comparator were realized simultaneously for two-input return-to-zero (RZ) signals at $40 \mathrm{Gbit} / \mathrm{s}$ on the basis of cross-gain modulation (XGM) and four-wave mixing (FWM) in three parallel SOAs [6], and a 2-to-4 level decoder was used to develop an all-optical two-input digital multiplexer based on cross polarization modulation in an SOA [7]. Sequential logic

*Corresponding author (email: xlzhang@mail.hust.edu.cn) circuits, such as an N-bit shift register [8] and binary counter [9], were designed and demonstrated exploiting ring buffers. On the other hand, logic minterms were reported $[10,11]$ for three-input demodulated differential phase-shift keying signals employing SOAs and delay interferometers (DIs) at 20 and $40 \mathrm{Gbit} / \mathrm{s}$ respectively, which can be considered a basic building block for arbitrary logic functions. However, to our best knowledge, a module that performs digital priority encoding in the optical domain has not yet been demonstrated.

It is known that a traditional digital binary encoder enables only one effective input at any one time. If more than one input is present at the same time, the circuit will miscode. However, in a practical situation, different inputs require responses simultaneously; e.g., the interrupt request in computer systems. A priority encoder allows the existence of multiple valid inputs simultaneously, identifies the priority of the request signals and encodes the priority.

In this paper, we demonstrate an all-optical 4-bit priority encoder operating at $40 \mathrm{Gbit} / \mathrm{s}$ based on XGM in SOAs. Detuning filters were employed after SOAs to improve dy- 
namic characteristics. The proposed scheme has the advantages of power efficiency, high operation speed and integration potential.

\section{Principle of operation}

The logic truth table for an all-optical digital 4-bit priority encoder is shown in Table $1 . I_{3}, I_{2}, I_{1}$ and $I_{0}$ are four line inputs, and the logic state " 1 " indicates that a response is required while the logic state " 0 " indicates that it is not. " $x$ " is an arbitrary item that could be " 1 " or " 0 ". $Y_{1}$ and $Y_{0}$ are the final outputs encoded according to the priority of the signals requiring a response. With regard to a stable system, the output is " 0 " if there is no input. Therefore, $Y_{1} Y_{0}$ is " 00 " if $I_{3} I_{2} I_{1} I_{0}=0000$. However, this conflicts with the output encoding when $I_{3} I_{2} I_{1} I_{0}=0001$. Thus, $G S$ is set to identify whether there are inputs, and it gives the system a warning output, logic state "1", if the input is 0000 . In this circumstance, although the output code is 00 , it is invalid. From the truth table, we also recognize that the priority order of the inputs from high to low is $I_{3}, I_{2}, I_{1}, I_{0}$. Therefore, if $I_{3}$ and $I_{2}$ are both " 1 ", only $I_{3}$ is encoded.

According to Table 1, the output expressions of the priority encoder are written as

$$
\left\{\begin{array}{l}
Y_{1}=I_{3}+I_{2}, \\
Y_{0}=I_{3}+\overline{I_{2}} I_{1}, \\
G S=\overline{I_{3}} \overline{I_{2}} \overline{I_{1}} \frac{I_{0}}{} .
\end{array}\right.
$$

From eq. (1), we see that the priority encoder is mainly made up of logic OR and AND functions. However, the OR logic contained in $Y_{1}$ and $Y_{0}$ cannot be obtained by coupling the items on the right of the expressions directly because there might be an identical bit " 1 " in different inputs that will induce multilevel intensities in the outputs $Y_{1}$ and $Y_{0}$. Apart from this, at least two SOAs are required to achieve $Y_{0}$. One is used to obtain $\overline{I_{2}} I_{1}$ and the other is used to obtain $I_{3}+\bar{I}_{2} I_{1}$. GS is also difficult to obtain using an AND gate because there are too many inputs. To avoid multilevel intensities, reduce the number of SOAs and avoid using AND

Table 1 Logic truth table for an all-optical digital 4-bit priority encoder

\begin{tabular}{ccccccc}
\hline & \multicolumn{2}{c}{ Input } & & \multicolumn{3}{c}{ Output } \\
\hline$I_{3}$ & $I_{2}$ & $I_{1}$ & $I_{0}$ & $Y_{1}$ & $Y_{0}$ & $G S$ \\
0 & 0 & 0 & 0 & 0 & 0 & 1 \\
0 & 0 & 0 & 1 & 0 & 0 & 0 \\
0 & 0 & 1 & $\times$ & 0 & 1 & 0 \\
0 & 1 & $\times$ & $\times$ & 1 & 0 & 0 \\
1 & $\times$ & $\times$ & $\times$ & 1 & 1 & 0 \\
\hline
\end{tabular}

logic for multiple inputs, the expressions are modified as

$$
\left\{\begin{aligned}
Y_{1} & =I_{3}+\overline{I_{3}} I_{2}, \\
Y_{0} & =I_{3}+\overline{I_{3}} \overline{I_{2}} I_{1} \\
& =I_{3}+\overline{\left(I_{3}+I_{2}\right)} I_{1}, \\
G S & =\overline{I_{3}+I_{2}+I_{1}+I_{0}} .
\end{aligned}\right.
$$

As shown in eq. (2), $Y_{1}$ and $Y_{0}$ are never multilevel because of the simultaneous existence of $I_{3}$ and $\overline{I_{3}}$, which cannot be " 1 " at the same time. Thus, $Y_{1}$ and $Y_{0}$ are easily realized by coupling the items on the right of the expressions directly. $\overline{I_{3}} I_{2} I_{1}$ (in $Y_{0}$ ) and $G S$ are modified to $\overline{\left(I_{3}+I_{2}\right)} I_{1}$ and $\overline{I_{3}+I_{2}+I_{1}+I_{0}}$ respectively because the realization of the NOR gate in the SOA is much easier than that of the AND gate.

The system configuration is illustrated in Figure 1. Regarding $Y_{1}$, the intensity of the probe light $I_{2}$ is modulated by the intensity of the pump light $I_{3}$ according to the XGM effect in the SOA. When $I_{3}$ is strong (high level), carriers in SOA1 are heavily consumed and SOA1 becomes saturated. Thus, $I_{2}$ cannot be amplified and the output is " 0 ". On the contrary, when $I_{3}$ is weak (low level), almost no carriers in SOA 1 are consumed and $I_{2}$ is amplified. Therefore, the logic operation $\bar{I}_{3} I_{2}$ is easily obtained at the output of SOA1 when the average power of $I_{3}$ is much greater than that of $I_{2}$. $Y_{1}$ can then be achieved by coupling $\overline{I_{3}} I_{2}$ and $I_{3}$ directly. As for $Y_{0}$, both $I_{3}$ and $I_{2}$ act as pump lights with nearly the same power, and $I_{1}$ serves as the probe light. With a similar operation principle, SOA2 becomes saturated as long as either $I_{3}$ or $I_{2}$ is high, and $I_{1}$ is suppressed accordingly. Only when both $I_{3}$ and $I_{2}$ are low is $I_{1}$ amplified. Hence, we obtain $\overline{\left(I_{3}+I_{2}\right)} I_{1}$, and $Y_{0}$ is demonstrated by combining $\overline{\left(I_{3}+I_{2}\right)} I_{1}$ and $I_{3}$. While the pump lights $I_{3}, I_{2}, I_{1}$ and $I_{0}$ are co-propagated with another continuous-wave $(\mathrm{cw})$ probe

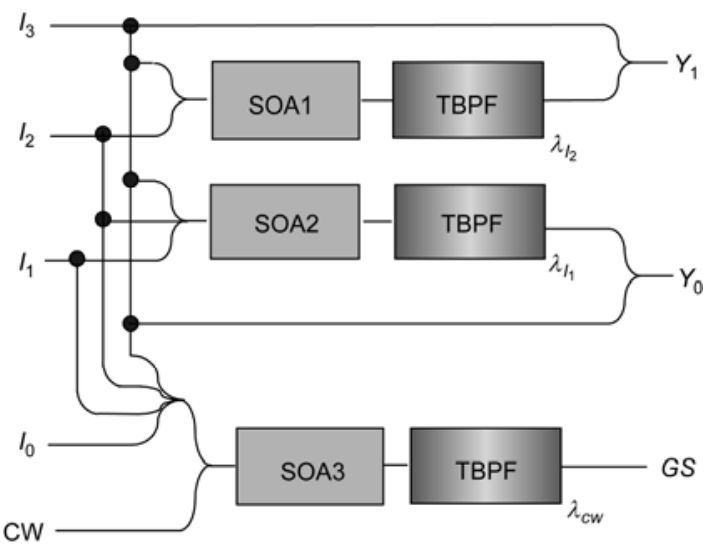

Figure 1 System configuration. 
light, the logic NOR gate for multiple inputs can be realized and the warning output $G S$ is achieved.

\section{Experimental setup and results}

The experimental setup for the all-optical digital 4-bit priority encoder is depicted in Figure 2. The cw beams generated by LD1, LD2, LD3, LD4 and LD5 have wavelengths of $1560.75\left(\lambda_{I_{3}}\right), 1557.69\left(\lambda_{I_{2}}\right), 1554.45\left(\lambda_{I_{1}}\right), 1551.24\left(\lambda_{I_{0}}\right)$ and $1547.72 \mathrm{~nm}\left(\lambda_{c w}\right)$, respectively. The data signals at wavelengths from $\lambda_{I_{3}}$ to $\lambda_{I_{0}}$ are modulated simultaneously by two Mach-Zehnder modulators (MZMs) to produce RZ signals at $40 \mathrm{Gbit} / \mathrm{s}$ with a duty cycle of $33 \%$. The fixed data stream "1001 001101010010 " is provided by the bit pattern generator (BPG). An erbium-doped fiber amplifier (EDFA) is exploited to amplify the signals to $15.63 \mathrm{dBm}$. The four data signals are then separated by a wavelength- division demultiplexer (WDM) with channel spacing of $1.6 \mathrm{~nm}$. The signals at $\lambda_{I_{2}}, \lambda_{I_{1}}$ and $\lambda_{I_{0}}$ are delayed with different bit time durations by optical delay lines (ODLs) to emulate four different data signals $I_{3}, I_{2}, I_{1}$ and $I_{0}$.

The corresponding absolute data sequences are "1001 00110101 0010", “0101 00100110 1010”, “0100 1010 01001101 ” and "1010 100101001001 ”. Their temporal waveforms and eye diagrams are recorded by the communication signal analyzer (CSA). The eye diagrams are measured for $2^{7}-1$ pseudo-random binary sequence input. $I_{3}$ is divided between two paths, one to combine with the SOA1 (CIP ultrafast SOA) output, and the other as one input of SOA1; the average powers are -6.12 and $10.35 \mathrm{dBm}$, respectively. $I_{2}$ is attenuated to $-8.96 \mathrm{dBm}$ as the probe light. SOA1 is biased at $250 \mathrm{~mA}$ with a recovery time of about 50 ps. The 3-dB bandwidth of the bandpass filter (TBPF) after SOA1 is $1 \mathrm{~nm}$, which ensures the pulse width of the output

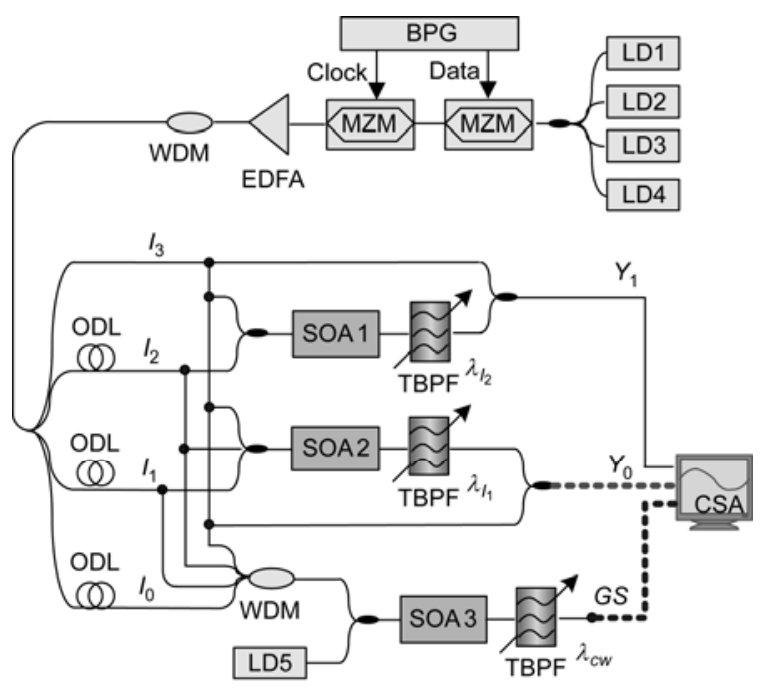

Figure 2 Experimental setup for the 4-bit all-optical digital priority encoder.
$\bar{I}_{3} I_{2}$ is not too wide. The TBPF central wavelength is detuned with a slight blue shift from $\lambda_{I_{2}}$ to improve the quality of the final logic signal and increase the operation speed. $Y_{1}$ is then achieved by coupling $\bar{I}_{3} I_{2}$ and $I_{3}$ directly.

Similarly, as for $\overline{\left(I_{3}+I_{2}\right)} I_{1}$, the average power of the SOA2 inputs, $I_{3}, I_{2}$ and $I_{1}$, are adjusted to $6.60,6.45$ and $-8.47 \mathrm{dBm}$, respectively. $Y_{0}$ is demonstrated by combining $\overline{\left(I_{3}+I_{2}\right)} I_{1}$ and $I_{3}$, and the TBPF is a little blue shifted from the signal light at $\lambda_{I_{1}}$. The cw light generated by LD5 serves as the probe light of SOA3 with power of $-7.72 \mathrm{dBm}$. The pump light is composed of $I_{3}, I_{2}, I_{1}$ and $I_{0}$, whose average powers are 5.27, 7.54, 10.12 and $8.99 \mathrm{dBm}$, respectively. The cw light is filtered out by the TBPF at the output port of SOA3, which provides the warning signal $G S$.

The temporal waveforms and eye diagrams are illustrated in Figure 3. Figures 3(a)-(d) show the original input data of the input signals. The zero levels of $\bar{I}_{3} I_{2}$ and $\overline{\left(I_{3}+I_{2}\right)} I_{1}$, as shown in Figure 3(e) and (f) respectively, are not so flat because the gain of the probe light is not compressed completely. The final output items $Y_{1}$ and $Y_{0}$ are depicted in Figure $3(\mathrm{~g})$ and $(\mathrm{h})$, respectively. Both are of higher quality than the intermediate results $\overline{I_{3}} I_{2}$ and $\overline{I_{3}} \bar{I}_{2} I_{1}$ since the original signal is used. The $G S$ output is shown in Figure 3(i).

Theoretically, GS should be a dark RZ format because the probe light is a continuous wave. However, owing to the heavy consumption of the carriers in the SOA when there are two, three or four " 1 " bits in the coupled input signals, as well as the carrier density recovery not being fast enough, a series of small ripples are present at the positions of " 0 " bits. For the same reason, the " 1 " bits, whose pulse width should be very wide, are suppressed by the next input pulse when they have not fully recovered. Consequently, the final output $G S$ is in the form of a RZ signal with a little broadening. Although the $G S$ data stream is not a perfect RZ signal, its temporal logic levels still satisfy the designed logic function.

Figure 4 shows the measured extinction ratios and eye opening factors of all logic units. All extinction ratios exceed $10 \mathrm{~dB}$, and the eye opening factors exceed 0.7. This scheme has the potential to operate at a higher bit rate because wavelength conversion with a similar program was demonstrated at $320 \mathrm{~Gb} / \mathrm{s}[12,13]$.

\section{Conclusion}

The all-optical digital 4-bit priority encoder based on RZ signals at $40 \mathrm{Gbit} / \mathrm{s}$ was proposed and experimentally demonstrated employing the XGM effect in SOAs cascaded with detuning bandpass filters. As the final results, correct and clear temporal waveforms and open eye patterns were 


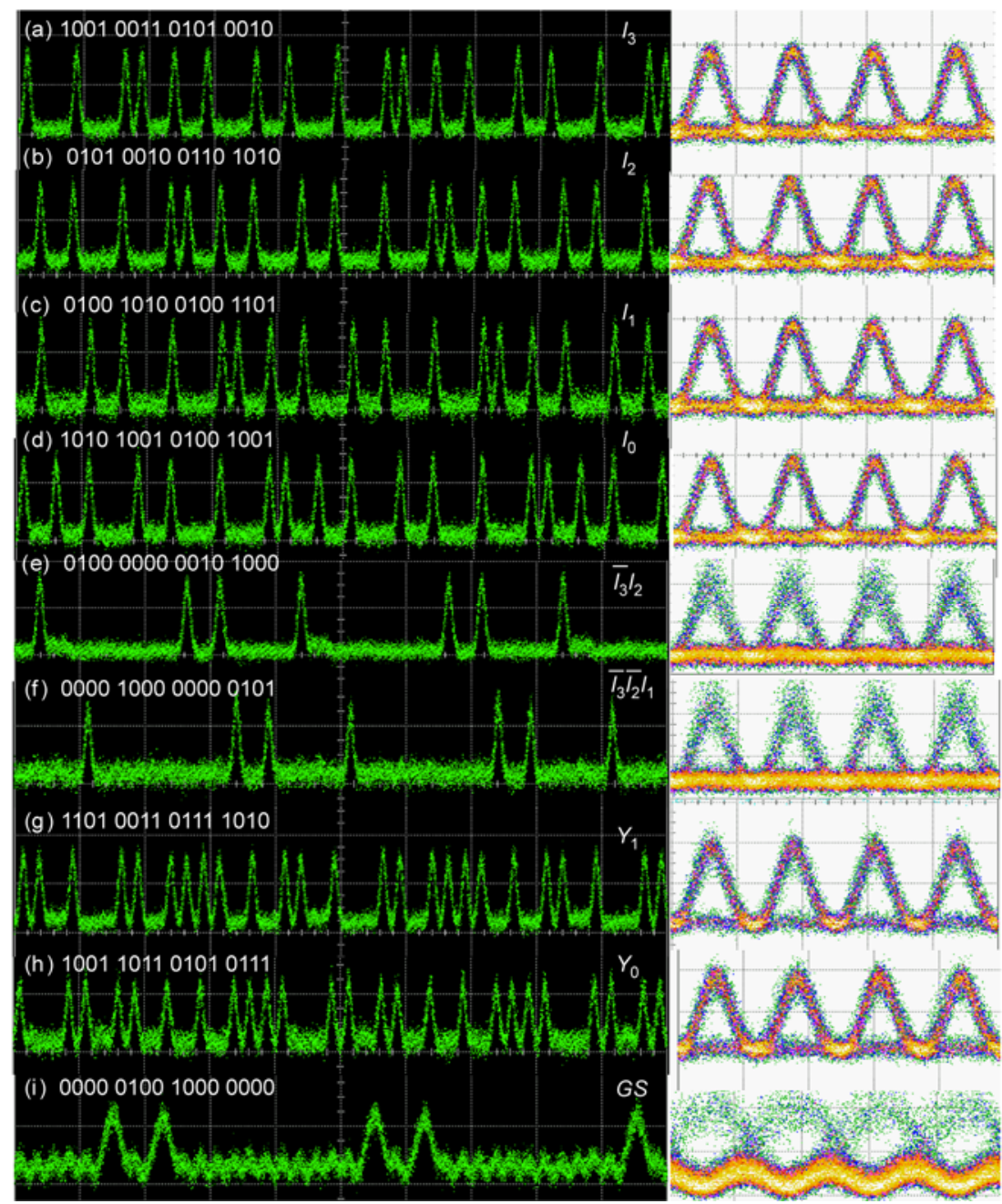

Figure 3 Temporal wave forms and eye diagrams of all logic units. (a)-(d) Original inputs; (e),(f) intermediate results; (g)-(i) final encoding outputs.

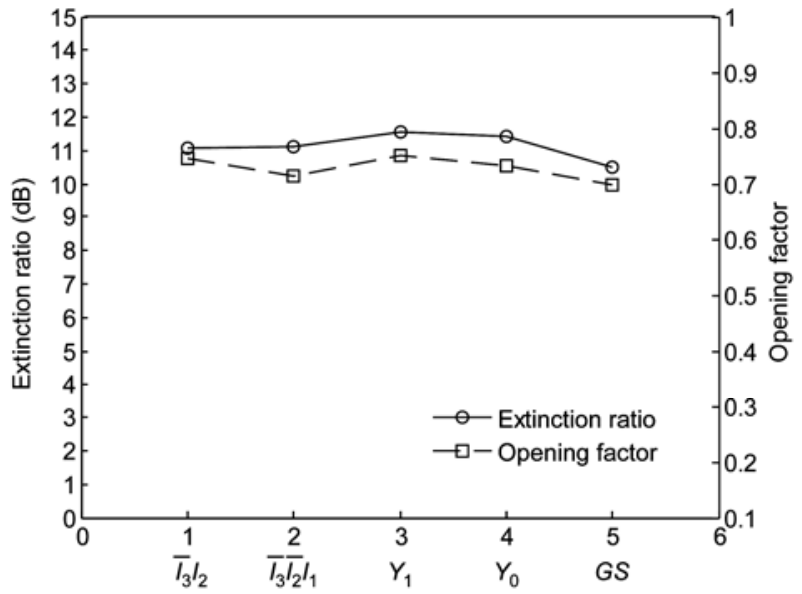

Figure 4 Extinction ratios and opening factors of all logic units.

obtained, and extinction ratios exceeding $10 \mathrm{~dB}$ were achieved. A more complicated digital priority encoder circuit with a higher operation bit rate could be realized by cascading the 4-bit priority encoder. Moreover, the proposed scheme has the potential to be large-scale integrated.

This work was supported by the National Basic Research Program of China (2011CB301704), the National Natural Science Foundation of China (60877056 and 60901006) and the Program for New Century Excellent Talents in University of China (NCET-04-0715).

1 Xu J, Zhang X L, Liu D M, et al. Ultrafast all-optical NOR gate based on semiconductor optical amplifier and fiber delay interferometer. Opt Express, 2006, 14: 10708-10713

2 Wang J, Sun J Q, Sun Q Z, et al. PPLN-based flexible optical logic AND gate. IEEE Photon Technol Lett, 2008, 20: 211-213

3 Qiu J F, Sun K, Rochette M, et al. Reconfigurable all-optical multilogic gate (XOR, AND, and OR) based on cross-phase modulation in a highly nonlinear fiber. IEEE Photon Technol Lett, 2010, 22: 1041-1135

4 Tsiokos D, Kehayas E, Vyrsokinos K, et al. 10-Gb/s all-optical half-adder with interferometric SOA gates. IEEE Photon Technol Lett, 2004, 16: 284-286

5 John S K, McGeehan E, Kumar S, et al. Simultaneous optical digital half-subtraction and addition using SOAs and a PPLN waveguide. Opt Express, 2007, 15: 5543-5549 
6 Wang Y, Zhang X L, Dong J J, et al. Simultaneous demonstration on all-optical digital encoder and comparator at $40 \mathrm{~Gb} / \mathrm{s}$ with semiconductor optical amplifiers. Opt Express, 2007, 15: 15080-15085

7 Soto H, Gutiérrez A. All-optical 2-to-4 level encoder based on cross polarization modulation in a semiconductor optical amplifier utilized to develop an all-optical 2 input digital multiplexer. Opt Express, 2006, 14: 9000-9005

8 Lazzeri E, Berrettini G, Meloni G, et al. All-optical n-bits shift register exploiting a ring buffer based on semiconductor optical amplifier. IEEE Photon Technol Lett, 2011, 23: 45-47

9 Wang J, Meloni G, Berrettini G, et al. All-optical binary counter based on semiconductor optical amplifiers. Opt Lett, 2009, 34: $3517-$ 3519

$10 \mathrm{Xu} \mathrm{J}$, Zhang X L, Dong J J, et al. All-optical minterm generator for three-input NRZ-DPSK signals based on SOAs and delay interferometers. In: Optical Fiber Communication Conference \& Exposition and the National Fiber Optic Engineers Conference, USA, San Diego, 2008. JWA78

11 Chen X B, Yu Y, Zhang X L. All-optical logic minterms for three-input demodulated differential phase-shift keying signals at 40 Gb/s. IEEE Photon Technol Lett, 2011, 23: 118-120

12 Liu Y, Tangdiongga E, Li Z, et al. Error-free $320 \mathrm{~Gb} / \mathrm{s}$ SOA-based wavelength conversion using optical filtering. In: Optical Fiber Communication Conference \& Exposition and the National Fiber Optic Engineers Conference, USA, California, 2006. PDP28

13 Liu Y, Tangdiongga E, Li Z, et al. Error-free 320-GB/s all-optical wavelength conversion using a single semiconductor optical amplifier. J Lightw Technol, 2008, 25: 103-108

Open Access This article is distributed under the terms of the Creative Commons Attribution License which permits any use, distribution, and reproduction in any medium, provided the original author(s) and source are credited. 